\title{
Genetic Variabilities and Genetic Gains for Yield Components in Regional Sugarcane Breeding Programmes on Réunion Island
}

\author{
Thomas Dumont $^{1} \cdot$ Audrey Thong-Chane $^{1} \cdot$ Laurent Barau $^{1} \cdot$ Bernard Siegmund $^{1}$ \\ Jean-Yves Hoarau ${ }^{1,2,3}$ (B)
}

Received: 2 November 2018/ Accepted: 9 March 2019/Published online: 20 March 2019

(C) The Author(s) 2019

\begin{abstract}
Sugarcane variety development programmes are costly and lengthy. It appears important to periodically assess their ability to select competitive genotypes for yield components and provide genetic gains. This article reports a 10-year retrospective analysis of successive variety trials conducted in four regional breeding programmes on Réunion Island in the most advanced regional selection stage. The four variety programmes were dedicated to the humid coastal zone (LM), the per-humid coastal zone (SB), the irrigated dry coastal zone (ES) and the dry high lands (VB) of the local industry. Using mixed linear models, the objective of the study was to (1) assess genetic variabilities available for yield components in this advanced selection stage; and (2) estimate trends of genetic gains achieved over the last decade across the four programmes. Yield components were: cane yield (CY), estimable recoverable sugar (ERS), fibre content (FIB) and economic index (EI). Broad-sense heritabilities were high $(0.70-0.91)$ for all traits in each programme. Mean genetic coefficients of variation were about twice as high for CY (14.1\%) and EI (15.5\%) compared to ERS (5.7\%) and FIB (6.6\%). A higher probability of identifying superior varieties was found for $\mathrm{CY}$ and EI in two of the four programmes characterized either by thermal and hydric stresses (VB) or by an edaphic stress (SB). Simple linear regression of
\end{abstract}

Jean-Yves Hoarau

hoarau.jean-yves@ercane.re

1 ERCANE, 29 rue d'Emmerez de Charmoy, 97490 Sainte-Clotilde, La Réunion, France

2 CIRAD, UMR AGAP, 97490 Sainte-Clotilde, La Réunion, France

3 Univ Montpellier, CIRAD, INRA, Montpellier SupAgro, Montpellier, France variety performances versus years of selection revealed trends in genetic gains for EI ranging between 0.53 and $1.81 \%$ increase per year that were highly significant $(P<0.001)$ in the two programmes (LM and VB).

Keywords Sugarcane · Yield components .

Mixed linear models $\cdot$ Breeding programmes

\section{Introduction}

Genetic improvement has played a major role in yield increase in the last decades in many sugarcane-producing regions around the world as illustrated by studies in Australia (Jackson 2005; Cox and Stringer 2007), South Africa (Zhou and Gwata 2016), Argentina (Acreche et al. 2015) or Florida (Edme et al. 2005) based on long-term analyses of commercial and/or trial productivity data. Although breeders take many traits into consideration, such as disease resistance, harvesting characteristics, ratooning ability, their top priority is to select for high sugar yield through its two components: cane tonnage and sugar content.

In Réunion Island, during the last four decades urban encroachment has left less land for cultivation so most of the improvement in productivity has come from yield increases rather than more acreage being planted. Sugarcane is cultivated all around the Island on both the wet windward coast and the dry leeward coast, from sea level up to $800 \mathrm{~m}$ of altitude. Therefore, production of sugarcane is scattered over many different regions in terms of soil types and climates due to variable rainfall, temperature and sunlight levels. In the coming years, the sustainability of cane production of Réunion Island will therefore depend on productivity gains that could be achieved in each agro- 
climatic region of the industry with the release of new and ever-higher yielding cultivars.

eRcane research institute which is responsible for the Sugarcane Variety Development Programme (SVDP) of Réunion Island currently operates seven decentralized concurrent selection programmes located in seven major growing areas (Guilly et al. 2017). The programme currently takes 14 years from the initial cross to the release of a new R-cultivar. Each year, about 100,000 new genotypes are obtained from true seeds coming from between 2000 and 2500 bi-parental genetic combinations. Progenies of all combinations are dispatched among the different programmes. The different materials tested in these regional programmes undergo, in parallel, the same sequential process of selection during four successive stages of selection (stages I-IV) that last a total of 10 years (Table 1). Highly significant genotype-by-environment interactions exist among the seven sites of selection for cane yield (CY) and estimable recoverable sugar (ERS), fibre content (FIB) and the derived economic index (EI) (Guilly et al. 2017). The establishment of these decentralized concurrent selection programmes was motivated by a strategy based on an early selection pressure for local adaptation. The objective was to increase the frequency of identification of superior candidates in each major area of the industry to enhance the chance of identification of new R-cultivars locally adapted, and ultimately to increase the productivity of the whole industry.

Four of the seven regional programmes are dedicated to the four major agro-climatic production zones of Réunion Island, namely the coastal humid and per-humid zones, the costal irrigated dry zone and the dry high lands (Fig. 1).
Considering the high costs and the long duration of these programmes, it appears important periodically to assess their ability to select competitive genotype populations for sugar yield components (CY, ERS, FIB and EI) and deliver genetic gains over time. Since 2007, ten successive series of varieties were tested in the most advanced stage of local selection (stage IV) in each programme. Based on this decade of trial data, the objective of this study was to assess in these regional programmes: (1) genetic variabilities available for yield components; (2) trends of genetic gains achieved over time; (3) discuss breeding strategies for improved sugar yield $(\mathrm{CY} \times \mathrm{ERS})$.

\section{Materials and Methods}

\section{Experimental Sites}

The four programmes are located in La Mare (LM), SaintBenoit (SB), Etang-Salé (ES) and Vue-Belle (VB) stations on Réunion Island in contrasted environments representative of the four major cultivation areas of sugarcane. Our previous paper (Guilly et al. 2017) gives detailed information on their respective locations, climate characteristics and soil types in terms of physical properties and chemical fertility. Briefly summarized, the sites of LM and SB are located on the wet windward coast of the island in its North and East regions, respectively; while the sites of VB and ES are located on the dry leeward coast of the island in its West and South regions, respectively. The SB programme (3290 $\mathrm{mm}$ of annual rainfall) is dedicated to the per-humid zone, the LM programme $(1470 \mathrm{~mm})$ to the humid zone,

Table 1 Progression of selection in the decentralized selection programmes of Réunion

\begin{tabular}{|c|c|c|c|c|c|c|c|c|}
\hline $\begin{array}{l}\text { Selection } \\
\text { stage }\end{array}$ & $\begin{array}{l}\text { No. of } \\
\text { decentralized } \\
\text { programmes }\end{array}$ & $\begin{array}{l}\text { Clones per } \\
\text { site/total }\end{array}$ & Trial design & $\begin{array}{l}\text { No. of } \\
\text { replicates }\end{array}$ & $\begin{array}{l}\text { No. } \\
\text { of } \\
\text { crops }\end{array}$ & $\begin{array}{l}\text { Select } \\
\text { rate } \\
(\%)\end{array}$ & \multicolumn{2}{|c|}{ Selection criteria } \\
\hline $\begin{array}{l}\text { Stage I } \\
\text { seedlings }\end{array}$ & 7 & $\begin{array}{l}15,000 \times 7 \\
105,000\end{array}$ & $\begin{array}{l}\text { Partial } \\
\text { replication } \\
\text { of families }\end{array}$ & & 1 & & \multicolumn{2}{|c|}{ Family values, visual assessment and freedom from diseases } \\
\hline $\begin{array}{l}\text { Stage II } \\
1 \text { row }\end{array}$ & 7 & $\begin{array}{l}1800 \times 7 \\
12,500\end{array}$ & Control plot & 1 & 2 & 12 & $\begin{array}{c}\text { Brix, visual } \\
\text { biomass }\end{array}$ & $\begin{array}{l}\text { Freedom from naturally occurring diseases } \\
\text { and pests, minimum flowering rate, rapid } \\
\text { germination growth, plant erectness }\end{array}$ \\
\hline $\begin{array}{l}\text { Stage III } \\
2 \text { rows }\end{array}$ & 7 & $\begin{array}{l}115 \times 7 \\
800\end{array}$ & $\mathrm{RCB}^{1}$ & 2 & 3 & 6.5 & \multirow{2}{*}{\multicolumn{2}{|c|}{$\begin{array}{l}\text { Cane yield (CY), estimable recoverable sugar (ERS), fibre } \\
\text { content (FIB), ratooning ability }\end{array}$}} \\
\hline $\begin{array}{l}\text { Stage IV } \\
3 \text { rows }\end{array}$ & 7 & $\begin{array}{l}25 \times 7 \\
175\end{array}$ & $\mathrm{RCB}^{1}$ & 3 & 3 & 22 & & \\
\hline $\begin{array}{l}\text { Stage V } \\
\text { multi- } \\
\text { local }\end{array}$ & & $\begin{array}{l}25 \times 1 \\
25\end{array}$ & $\mathrm{RCB}^{1}$ & 4 & 4 & 14 & $\begin{array}{l}\text { Wider } \\
\text { potential } \\
\text { zones of } \\
\text { adaptation }\end{array}$ & $\begin{array}{l}\text { Inoculated-based disease resistance tests } \\
\text { (smut, leaf scald and gumming) }\end{array}$ \\
\hline
\end{tabular}

$R C B$ randomized complete blocks 


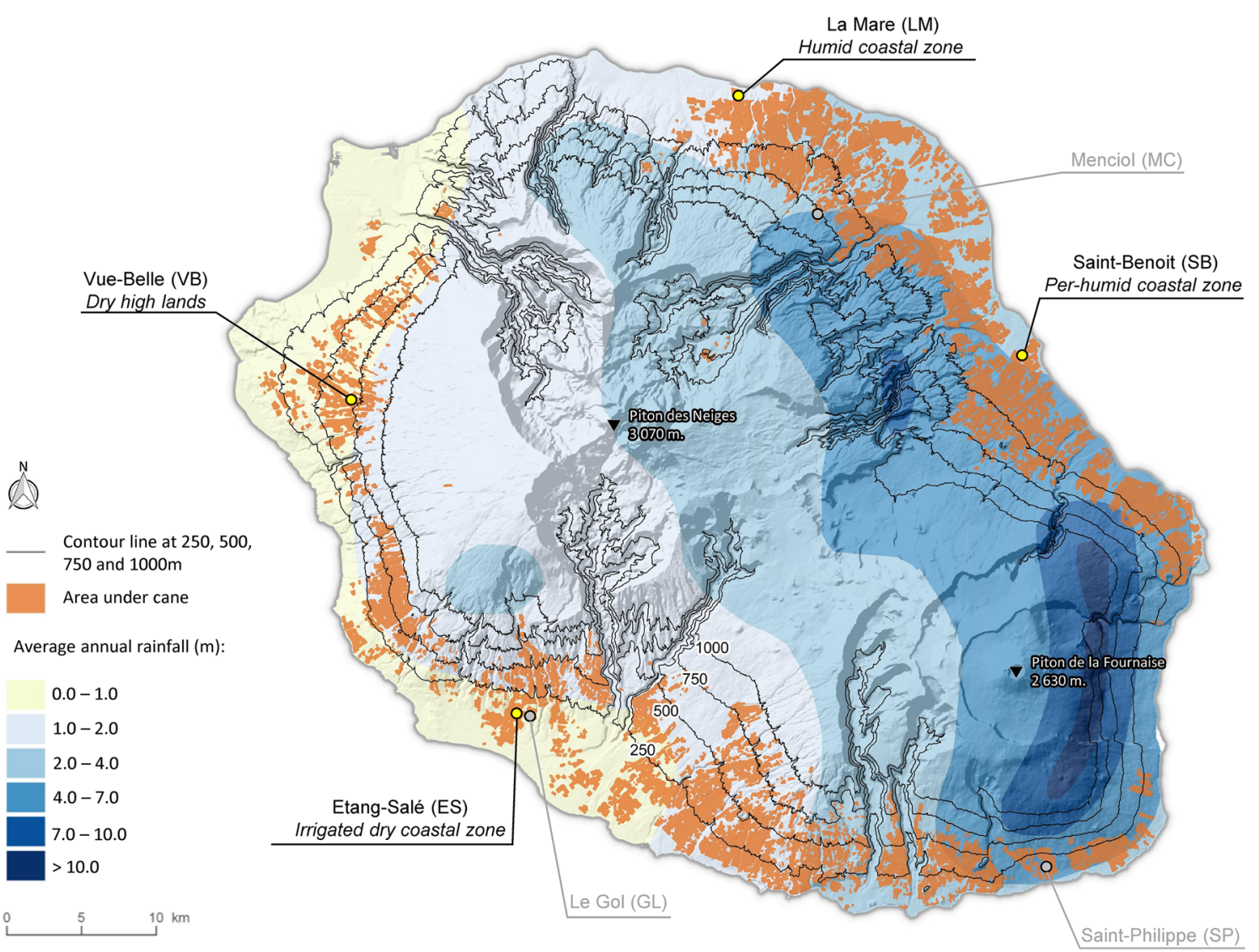

Fig. 1 Location of the different regional sugarcane selection programmes in Réunion Island

the VB programme $(870 \mathrm{~mm})$ to the dry high land and the ES programme $(614 \mathrm{~mm})$ to the irrigated dry zone. Trials in SB and VB are conducted in rainfed cropping conditions. The VB site suffers from a rainfall deficit during 7 months (from May to November). Trials in LM and ES are irrigated to compensate the water deficit of these sites. The sites of LM, SB and ES are at relatively low altitudes above sea level synonymous with high mean temperatures throughout the year (ranging from 20 to $27^{\circ} \mathrm{C}$ ) favourable for cane growth. On the contrary, the lower range of mean temperature $\left(14.5-20{ }^{\circ} \mathrm{C}\right)$ at the high altitude of the VB site (700 $\mathrm{m}$ above sea level) implies a seasonal thermal stress in the "winter season" which is less favourable for plant growth. The soil classification (ISSS, 1998) of the sites, their physical properties and the depth of the topsoil layer distinguish in particular SB from the other three sites. SB (Hydric Andisol) has a very stony soil and a relatively fine layer of topsoil ( $0.25 \mathrm{~m}$ on average). As a result, the soil dries quickly after rain and its water reserve available for the plant is small. On the contrary, the other three stations are not (LM, Nitisol; VB, Umbrisol) or only slightly (ES) stony and have deeper topsoil layers $(0.50-1.00 \mathrm{~m}$ or more). Compared with SB, these soil characteristics make water available to the plant over lengthier periods after either rain (VB) or irrigation (ES and LM).

\section{Variety Trials}

Ten different series of variety trials were successively planted in stage IV between 2004 and 2013 in each of the four programmes in replicated trials consisting of randomized complete block (RCB) designs with three replicates. The individual variety plot was $45 \mathrm{~m}^{2}$ (three rows of $10 \mathrm{~m}$ separated by $1.5 \mathrm{~m}$ ). Each trial was harvested and phenotyped in plant, first and second ratoon crops between 2005 and 2016. For example, trials planted in 2008 were harvested and phenotyped in 2009, 2010 and 2011 in the plant, first and second ratoon crops, respectively. The ten series were selected in second ratoon crop between 2007 and 2016. An average of 25 genotypes per series were tested at stage IV in each programme. Each series of varieties came from within the selection site where they had undergone all their previous selection steps (Table 1). The cultivar R579 was planted as a long-term standard in all trials of the LM, SB and ES programmes, while the cultivar R577 was the long-term standard in all trials of VB programme. The first cultivar is popular in wet $(\mathrm{LM}, \mathrm{SB}$, 
SP) or irrigated (ES) coastal areas, while the second cultivar is popular in highland areas (VB). The cultivars R579 (Corcodel et al. 2011) and R577 have the specific characteristic of relatively high sucrose content due to a fibre content which is always remarkably low. In addition, several other cultivars which were released more recently were also planted as standards: R582 in the trials of LM, SB and ES from 2007 and beyond; R583 in the trials of VB from 2008 and beyond and R585 in the trials of LM from 2007 and beyond.

\section{Yield Components}

The traits which were measured in all trials at individual plot level in each crop-year were: cane yield (CY, $\mathrm{Mg} \mathrm{ha}^{-1}$ ), fibre content as percentage of fresh weight (FIB, $\%$ ), estimable recoverable sugar (ERS, \%) and an economic index (EI). All millable stalks from each variety plot were manually cut using a hand-held cane knife and weighed using a digital scale mounted on a tractor operated hydraulic boom. From each plot, a sample of 18 randomly chosen stalks was used to determine ERS and FIB at eRcane laboratory. Trials harvested between 2005 and 2014 were assessed using the hydraulic press method (Hoarau 1969): FIB was determined from the weight of the press cake from a 500-g shredded subsample. ERS was calculated from the FIB value and Brix (Bellingham RFM340 refractometer) and Pol (REI Polaser SR polarimeter) measurements of the extracted juice according to conventional calculations used in the local cane industry adapted from Saranin (1986). ERS and FIB of trials harvested from 2014 and beyond were assessed with a new laboratory eRcane protocol using a semi-automatic nearinfrared spectroscopy (NIRS) device (Bruker Optik GmbH) and in-house calibrations; with a coefficient of determination $\left(\mathrm{R}^{2}\right)$ between predicted and observed values exceeding 0.9 and a ratio performance to deviation ( $\mathrm{RPD}=$ standard deviation/standard deviation of residuals) higher than 3 for ERS trait and a $R^{2}>0.7$ and a RPD $>2$ for FIB trait (Roussel et al. 2015). EI trait used by eRcane to rank cultivation merit of genotypes was calculated from $\mathrm{CY}$ and ERS using the formula $\mathrm{EI}=\mathrm{CY} \times(\mathrm{ERS}-4)$, which is indicative of the farming profit net of production costs (Hugot 1958).

\section{Statistical Analyses}

\section{Individual Trial Analysis}

The 40 trials which consisted of RCB designs were analysed individually with the following mixed linear model fit to each trait:
$Y_{i j k}=\mu+G_{i}+R_{j}+C_{k}+\mathrm{GC}_{i k}+e_{i j k}$

(Model 1)

where $Y_{i j k}$ designated the observation of genotype $i$ in replicate $j$ in crop-year $k$; $\mu$ the grand mean, $G_{i}$ the random main effect of $i$ th genotype, $R_{j}$ the fixed effect of the $j$ th replication, $C_{k}$ the fixed effect of the $k$ th crop-year, $G C_{i k}$ the random interaction effect between the $i$ th genotype and the $k$ th crop-year and $e_{i j k}$ the residual error. The model was run with the MIXED procedure of SAS 9.3 (SAS Institute, Cary, NC), and variance component estimates were evaluated using the restricted maximum likelihood (REML) procedure. Measurements acquired on the same individual plot across successive crop-years were considered as repeated measurements (longitudinal data) with an unstructured option (UN) for the matrix of variance-covariance of random error terms (Littell et al. 2006).

Broad-sense heritability $(H)$ at trial level for each trait was calculated with variance components estimated from the model, as the ratio of the genetic variance $\left(\sigma_{G}^{2}\right)$ to the phenotypic variance of genotype means $\left(\sigma_{P}^{2}\right)$ :

$H=\frac{\sigma_{G}^{2}}{\sigma_{P}^{2}}$

where $\sigma_{P}^{2}=\sigma_{G}^{2}+\frac{\sigma_{\mathrm{GC}}^{2}}{C}+\frac{\sigma_{e}^{2}}{\mathrm{CR}}$ with $\sigma_{\mathrm{GC}}^{2}$ and $\sigma_{e}^{2}$ designating GC interaction variance and mean error variance over cropyears, respectively; and considering $R=3$ replicates and $C=3$ crop-years.

Genetic coefficients of variation $\left(\mathrm{CV}_{\mathrm{g}} \%\right)$ were calculated for each trait and trial by dividing the corresponding genetic variance $\left(\sigma_{G}^{2}\right)$ by trial mean. Subsequently, mean $\mathrm{CV}_{\mathrm{g}}$ values across the ten local $\mathrm{R}$-series were compared between traits and programmes with a Duncan's multiplerange test (Steel and Torrie 1980) using R Agricolae package (De Mendiburu 2014).

For each trial, bivariate analyses were performed to compute the genetic correlation $\left(\rho_{G}\right)$ between traits defined as follows:

$\rho_{G}=\frac{\sigma_{G 1 G 2}}{\sqrt{\sigma_{G 1}^{2} \sigma_{G 2}^{2}}}$

where $\sigma_{G 1 G 2}$ was the genetic covariance between traits and $\sigma_{G 1}^{2} \sigma_{G 2}^{2}$ the product of their genetic variance estimates (Falconer and MacKay 1996). For each genetic correlation, an approximate 99\% confidence interval (CI) was estimated based on \pm 2.57 times its standard error (Lynch and Walsh 1998) and the correlation was declared significantly different from zero at $P<0.01$ if the approximate 99\% CI did not include zero (Holland 2006).

\section{Performance of Successive R-Series Within Programmes}

To get an overview of performance of the successive variety series in each programme, a global analysis of the 
ten local R-series was performed, using the following linear mixed model which was fitted with each trait:

$$
\begin{aligned}
Y_{i j k m}= & \mu+S_{m}+G_{i}+R_{j(m)}+C_{k(m)}+\mathrm{GC}_{i k}+\mathrm{GCS}_{i k m} \\
& +e_{i j k m} \quad(\text { Model } 2)
\end{aligned}
$$

where $S_{m}$ designated the main random effect of the $m$ th R-series within each site, while other terms and indices were the same as in Model 1. In this model, genotype $\left(G_{i}\right)$ was considered as fixed effect since candidate genotypes were deliberately tested for their local performance and represented a variable of intrinsic interest; while replicate $\left(R_{j(m)}\right)$ and crop-year $\left(C_{k(m)}\right)$ were considered as random effects to allow inferences on genotypes which are not restricted to the observed field nor to the observed testing crop-years. The first effect $\left(R_{j(m)}\right)$ was regarded as a random sample from a population of experimental units within each programme, and the second effect $\left(C_{k(m)}\right)$ was regarded as a random sample from a population of confounded effects of crops and years (Kang et al. 1987). The models were run with the MIXED procedure of SAS 9.3 (SAS Institute, Cary, NC) using the REML procedure. In each variety programme, a variance-covariance matrix of errors (R) was modelled for each of the ten series by using the option GROUP = series in the REPEATED statement. A variance component TYPE for this $\mathbf{R}$ matrix was chosen to minimize the number of variance parameters.

In each site and trait, the adjusted mean $\left(\bar{Y}_{i . . m}\right)$ of genotypes across the tested crop-years of each $m$ series was obtained by adding the overall trait mean $(\mu)$ of Model 2 to the best linear unbiased estimate (BLUE) of genotypes (Littell et al. 2006). For easier comparisons of variety results between programmes across traits, the adjusted mean of genotypes $\left(\bar{Y}_{i . . m}\right)$ was divided by the adjusted mean of the long-term commercial standard of each programme (R579 in LM, SB and ES or R577 in VB). The genotype means ( $y$-axis) were plotted against the series years ( $x$-axis) in each site. Finally, in order to determine the significance of the genetic gains of each trait within the decade period of selection (2007-2016), a regression analysis was performed using the REG procedure of SAS where genotype means (\% standard) was the response variable and the series was the predictor variable.

\section{Results}

\section{Genetic Parameters of Traits at Trial Level}

Table 2 summarizes mean values of broad-sense heritability $(H)$ and genetic coefficient of variation $\left(\mathrm{CV}_{\mathrm{g}}\right)$ of traits at experiment level. Mean $H$ values across programmes, which are a direct indicator of the effectiveness
Table 2 Mean broad-sense heritability $(\mathrm{H} \% \pm \mathrm{SE})$ and mean genetic coefficient of variation $\left(\mathrm{CV}_{\mathrm{g}} \% \pm \mathrm{SE}\right)$ at trial level in each programme for cane yield (CY), estimable recoverable sugar (ERS), fibre content (FIB) and economic index (EI) across ten successive variety series

\begin{tabular}{llll}
\hline Trait & Program & $\mathrm{H} \pm \mathrm{SE}$ & $\mathrm{CV}_{\mathrm{g}} \pm \mathrm{SE}^{\dagger}$ \\
\hline CY $\left(\mathrm{Mg} \mathrm{ha}^{-1}\right)$ & LM & $0.81 \pm 0.03$ & $13.7^{\mathrm{bc}} \pm 1.2$ \\
& SB & $0.80 \pm 0.03$ & $15.6^{\mathrm{ab}} \pm 1.9$ \\
& ES & $0.75 \pm 0.04$ & $11.3^{\mathrm{c}} \pm 1.1$ \\
& VB & $0.79 \pm 0.04$ & $15.8^{\mathrm{ab}} \pm 1.5$ \\
ERS $(\%)$ & Mean & 0.79 & 14.1 \\
& LM & $0.80 \pm 0.02$ & $4.4^{\mathrm{d}} \pm 0.4$ \\
& SB & $0.79 \pm 0.03$ & $5.1^{\mathrm{d}} \pm 0.2$ \\
& ES & $0.74 \pm 0.05$ & $5.8^{\mathrm{d}} \pm 0.7$ \\
FIB $(\%)$ & VB & $0.85 \pm 0.02$ & $7.6^{\mathrm{d}} \pm 0.8$ \\
& Mean & 0.79 & 5.7 \\
& LM & $0.91 \pm 0.02$ & $6.8^{\mathrm{d}} \pm 0.5$ \\
& SB & $0.88 \pm 0.02$ & $6.0^{\mathrm{d}} \pm 0.4$ \\
& ES & $0.87 \pm 0.02$ & $6.5^{\mathrm{d}} \pm 0.4$ \\
& VB & $0.87 \pm 0.02$ & $6.9^{\mathrm{d}} \pm 0.4$ \\
EI & Mean & 0.88 & 6.6 \\
& LM & $0.81 \pm 0.03$ & $15.2^{\mathrm{ab}} \pm 1.4$ \\
& SB & $0.82 \pm 0.01$ & $17.1^{\mathrm{a}} \pm 0.9$ \\
& ES & $0.74 \pm 0.05$ & $14.5^{\mathrm{ab}} \pm 1.0$ \\
& VB & $0.70 \pm 0.06$ & $15.4^{\mathrm{ab}} \pm 1.6$ \\
& Mean & 0.77 & 15.5 \\
\hline
\end{tabular}

$L M$ La Mare, $S B$ Saint-Benoit, ES Etang-Salé, $V B$ Vue-Belle

${ }^{\dagger}$ Values followed by same letter in the columns for each trait across all stages and sites are not significantly different at $P=0.05$

of the design of the experiments for selection decisionmaking, were very high for FIB (0.88) and to a lesser extent for CY (0.79) and ERS (0.79) and slightly less strong for the derived EI (0.77). $H$ values differed little between programmes for CY, ERS and FIB (<0.09), suggesting good repeatability for these traits.

Mean genetic coefficients of variation $\left(\mathrm{CV}_{\mathrm{g}} \%\right)$ spanned over a [11.3-15.8\%] range for $\mathrm{CY}$ among programmes and over a similar [14.5-17.1\%] range for EI. These ranges exceeded significantly $(P<0.05)$ the rather modest and very similar mean $\mathrm{CV}_{\mathrm{g}}$ ranges of ERS ([4.4-7.6\%]) and FIB ([6.0-6.9\%]). Mean $\mathrm{CV}_{\mathrm{g}}$ of $\mathrm{CY}$ was significantly $(P<0.05)$ larger in SB $(15.6 \%)$ and in VB $(15.8 \%)$ compared to ES (11.3\%). Mean $\mathrm{CV}_{\mathrm{g}}$ of ERS, FIB and EI was statistically similar across the four programmes.

Table 3 summarizes the mean values of genetic correlations between traits across the ten R-series tested in each of the four programmes (40 trials). The genetic correlation between $\mathrm{CY}$ and ERS was significant $(P<0.01)$ in only $15 \%(6 / 40)$ of the analyses and showed mean values almost null in LM (0.06), SB (-0.08) and ES (- 0.02) and slightly negative in VB $(-0.41)$. The genetic correlation 
Table 3 Genetic correlations between traits at trial level across ten R-series (above diagonal) and number of significant $(P<0.01)$ correlations (below diagonal) for cane yield (CY), estimable recoverable sugar (ERS), fibre content (FIB) and economic index (EI) in the four programmes

\begin{tabular}{|c|c|c|c|c|c|c|c|c|c|c|c|c|c|c|c|c|}
\hline \multirow{2}{*}{$\begin{array}{l}\text { Sites } \\
\text { Traits }\end{array}$} & \multicolumn{4}{|l|}{ LM } & \multicolumn{4}{|l|}{ SB } & \multicolumn{4}{|l|}{ ES } & \multicolumn{4}{|l|}{ VB } \\
\hline & $\mathrm{CY}$ & ERS & FIB & EI & $\mathrm{CY}$ & ERS & FIB & EI & $\mathrm{CY}$ & ERS & FIB & EI & $\mathrm{CY}$ & ERS & FIB & EI \\
\hline $\mathrm{CY}$ & - & 0.06 & -0.01 & 0.90 & - & -0.08 & 0.16 & 0.88 & - & -0.02 & -0.09 & 0.70 & - & -0.41 & 0.08 & 0.61 \\
\hline ERS & $(0)$ & - & -0.36 & 0.41 & (2) & - & -0.20 & 0.30 & (1) & - & -0.13 & 0.62 & (3) & - & -0.23 & 0.41 \\
\hline FIB & (1) & (5) & - & -0.16 & (1) & (1) & - & 0.06 & (1) & (1) & - & -0.20 & (1) & $(0)$ & - & -0.05 \\
\hline EI & (10) & (3) & $(0)$ & - & (10) & (5) & $(0)$ & - & (9) & (7) & (2) & - & (8) & (6) & (1) & - \\
\hline
\end{tabular}

$L M$ La Mare, SB Saint-Benoit, ES Etang-Salé, VB Vue-Belle

between CY and FIB was significant $(P<0.01)$ in $10 \%(4 /$ 40 ) of the analyses, and mean values in each site were low since they ranged between -0.09 and 0.16 . Mean genetic correlation between ERS and FIB quality traits was always slightly negative (between -0.36 and -0.13 ), but this correlation was significant $(P<0.01)$ in only $20 \%(7 / 40)$ of the analyses. Due to its derived calculation from CY and ERS, EI showed a high genetic correlation with $\mathrm{CY}$ ranging between 0.61 and 0.90 among sites; and a moderate-tohigh genetic correlation with ERS ranging between 0.30 and 0.62 depending on sites. The first (between CY and EI) and the second (between ERS and EI) correlations were significant $(P<0.01)$ at a frequency of $93 \%(37 / 40)$ and $53 \%$ (21/40), respectively.

\section{Performance of Successive R-Series Within Programmes}

Candidate varieties tested at stage IV have undergone a local process of selection in the specific environmental conditions of their programme since their initial seedling stage. Figure 2 shows variety performances of the ten successive local R-series of stage IV (model 2) as a relative percentage of a long-term standard (reference of 100\%) in each programme. The average [min-max] range across the four programmes was [74-116\%] for ERS, [90-143\%] for FIB, [42-140\%] for CY and [37-134\%] for EI. In all four programmes, the distribution pattern of varieties is systematically above the reference standards for the FIB and generally below the standards for the ERS.

\section{Humid Coastal Zone: LM Program}

CY of genotypes increased by $1.63 \%$ per year from 2007 to 2016 in the LM programme. This trend represented a highly significant correlation $(r=0.28, \quad P<0.001)$ between $\mathrm{CY}$ and year of selection. Genotype performances were in a $41-122 \%$ range of the control. Cane yield of the best genotypes increased from $96 \%$ in 2007 to $122 \%$ of the control by 2016. Although oriented upwards, trends in gains for ERS $(0.21 \%$ per year) and for FIB $(0.15 \%$ per year) were not significant $(P>0.05)$. EI had a $1.81 \%$ per year increase with advancing genotype series. This trend represented a highly significant correlation $(r=0.29$, $P<0.001)$ between EI and year of selection. From 2007 to 2012, only three genotypes showed higher EI than the control, but the programme produced 23 genotypes higher than the control from 2013 and onwards, among which three elite genotypes almost reaching $120 \%$.

\section{Per-humid Coastal Zone: SB Programme}

In the SB programme, CY showed a significant $0.88 \%$ increase per year with advancing genotype series $(r=0.13$, $P=0.042)$. Genotype performances for CY spanned over a large [45-151\%] range around the standard. In a decade, the program produced 19 genotypes with $\mathrm{CY}$ higher than $120 \%$ of the control among which three genotypes exceeded $140 \%$. ERS had a non-significant $-0.21 \%$ per year decrease, and FIB had a non-significant $0.15 \%$ per year increase $(P>0.05)$. EI showed an increasing trend of $0.78 \%$ per year which is almost statistically significant ( $r=0.12, P=0.072$ ). However, since 2012 the programme has produced 17 candidate genotypes that exceeded the control for EI by between $120 \%$ and $140 \%$.

\section{Irrigated Dry Coastal Zone: ES Programme}

The ES programme showed a highly significant $-1.76 \%$ decrease per year for $\mathrm{CY}$ among the local series tested at stage IV across the decade $(r=0.38, P<0.001)$. Genotype performances varied only in a $53-115 \%$ range. Candidate genotypes with $\mathrm{CY}$ values higher than that of the control were therefore rare, and their frequency decreased continuously. However, ERS showed a significant $(r=0.15, P=0.02)$ increase of $0.45 \%$ per year resulting in a non-significant $(r=0.10, P=0.147)$ increase of $0.53 \%$ per year for EI. Over the decade, only one candidate exceeded the control by $120 \%$ for EI. 


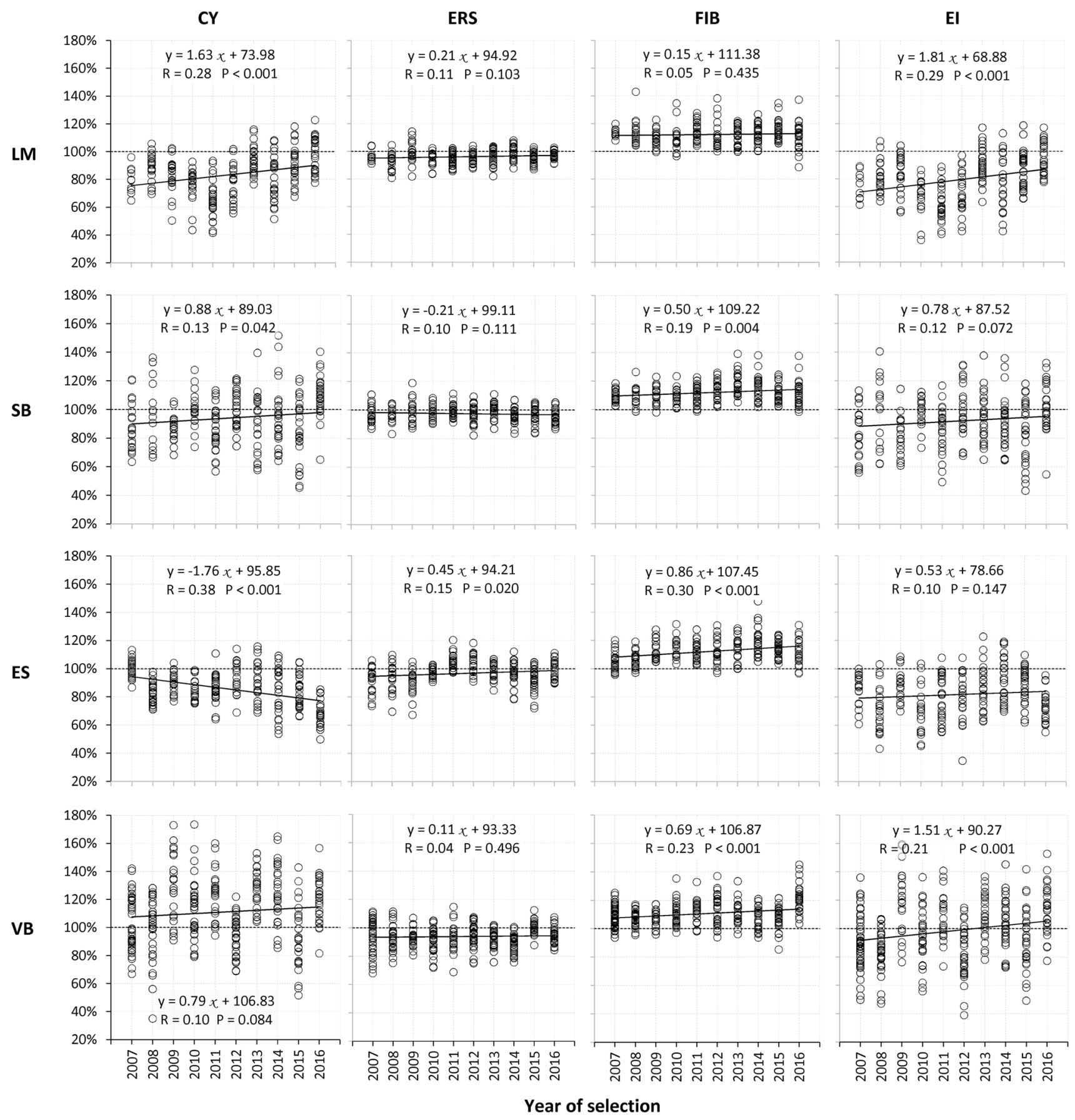

Fig. 2 Genetic variations and trends in gains for cane yield (CY), estimable recoverable sugar (ERS), fibre content (FIB) and economic index (EI) in four eRcane regional programmes between 2007 and
2016 (variety performances are expressed as a percentage of a longterm standard). $L M$ La Mare, $S B$ Saint-Benoit, ES Etang-Salé, VB Vue-Belle

$60 \%$ of the control for CY, while 31 genotypes showed a CY over $140 \%$. Gains in ERS showed a flat trend over the decade ( $r=0.04, P=0.496)$, while FIB trends showed a highly significant $0.69 \%$ increase per year $(r=0.23$, $P<0.001)$. Performances of genotypes for EI varied over a $38-159 \%$ range. In the last decade, the VB programme 
produced 13 candidates showing an EI less than $60 \%$ of the control and 49 candidates with EI over 120\%, among which seven were over $140 \%$.

\section{Discussion}

\section{Potential for Identifying Superior Cultivars}

The average [min-max] range, across all four programmes, of variety performances tested between 2007 and 2016 (Fig. 2) showed a larger amount of variation compared to the local controls for CY ([42-140\%]) and EI ([37-134\%]) than that found for both ERS ([74-116\%]) and FIB ([90-143\%]). Mean $\mathrm{CV}_{\mathrm{g}}$ at trial level (Table 2) was about twice as high for CY (14.1\%) and EI (15.5\%) compared to ERS (5.7\%) and FIB (6.6\%). Such large differences in genetic variation between yield (CY and EI) and quality (ERS and FIB) traits have also been found in highly selected (Brown and Glaz 2001; Tena et al. 2016) and unselected (Kang et al. 1990; Neto et al. 2013; Baffa et al. 2014) sugarcane populations. This narrow genetic variation for both ERS and FIB quality traits results from the fact that high-sugar and low-fibre parents are mainly used in our and other breeding programmes that are dedicated to industries producing sugar and first-generation bioethanol. Besides, the populations of stage IV we studied have undergone an earlier selection pressure in the previous stages (Table 1) on sucrose content and indirectly on fibre content (Table 3) compared to cane yield.

Variety performances for $\mathrm{CY}$ showed a larger amount of variation compared to the control in the SB ([45-151\%]) and VB ([35-175\%]) programmes compared to that found in the LM ([41-122\%]) and ES ([49-115\%]). At VB in the highlands, the context of combined thermal and water stresses during the cool dry season involves particular selection pressures. Hence, this higher variability of genotypes for cane yield in VB can be interpreted as more contrasted responses of candidate genotypes in VB programme than in LM and ES programmes due to these two particular stresses. The higher genetic variability in the SB programme for cane yield compared with LM and ES programmes is less obviously interpretable than in VB. However, the SB site is differentiated mainly from the other two sites (LM and ES) by non-irrigated conditions (rainfed crops) and stony, shallow soil (fine layer of topsoil of $0.25 \mathrm{~m}$ on average) resulting in lower water holding capacity, and therefore, less water is available for the plant during the dry season (May-October). One possible explanation of the higher genetic variability for cane yield in SB (compared with LM and ES) could reside in higher selection pressure related to some tolerance to drought and shallow soil; implying screening for more hardy varieties.
Conversely, the lower genetic variability of the cane yield in LM and ES could be related to more favourable environments for cane growth (absence of water stress due to irrigation practices combined with deeper fertile soils).

Figure 2 also shows that in the stage IV, the performance distribution of varieties for $\mathrm{CY}$ and $\mathrm{EI}$ in $\mathrm{SB}$ and VB is better centred on the commercial standard (100\%) than in LM and ES. These better-centred distributions illustrate that the standard R579 (which is a successful commercial cultivar in Réunion Island and in about ten other industries located in African, Caribbean and Asian sugar-producing countries) is still very competitive in humid (LM) and irrigated dry (ES) regions compared to new candidate genotypes currently under test. Moreover, Fig. 2 shows that the frequency of candidate genotypes exceeding $105 \%$ of the control for the sucrose content is rare; unlike the cane yield where, in some variety series, there are candidates exceeding $120 \%$ or more of the control. This illustrates that in the current breeding material, it is easier to select improved varieties on cane yield than on sucrose content. In the VB programme and to a lesser extent in the SB programme, the frequency of candidates over $120 \%$ of the control for CY and EI is high in stage IV, and much rarer in LM and ES programmes. These results indicate higher and more rapid chances of identifying new cultivars in the subsequent multi-local stage and in semi-commercial tests for per-humid (SB) zones or dry highland (VB) environments compared with humid (LM) zones and irrigated dry (ES) zones. Moreover, the average performance of the successive series for cane yield (CY) and economic index (EI) shows significant fluctuations from one series to another in each programme. Therefore, the statistical chances of obtaining superior cultivars adapted to each zone appear uneven from one year to another. These fluctuations of performances of candidates for CY and EI from a local series to another in each programme may result from two combining explanations: (1) an uneven genetic potential value for CY and EI between successive candidate populations and (2) selection efficiencies possibly uneven between series along previous selection stages I-III (because of trial designs necessarily less robust than in stage IV to assess CY and EI).

However, regressions of CY and EI over series in stage IV over the 2007-2016 period are interestingly oriented upwards in all four programmes, except in the dry irrigated programme (ES) for CY. Trend of genetic progress in LM, $\mathrm{SB}$ and $\mathrm{VB}$ programmes ranged for $\mathrm{CY}$ from 0.79 to $1.63 \%$ per year and from 0.79 to $1.81 \%$ per year for EI. All genetic trends found in this study higher than $0.88 \%$ per year (CY in SB) and higher than $1.51 \%$ per year (EI in VB and $\mathrm{LM}$ and $\mathrm{CY}$ in $\mathrm{LM}$ ) corresponded to significant $(P<0.05)$ or highly significant $(P<0.001)$ correlations between traits and selection years, respectively. These 
findings illustrate the potential of eRcane breeding programmes to produce genotypes that deliver genetic gains over time for $\mathrm{CY}$ and $\mathrm{EI}$.

Selection for increased fibre levels (FIB) is not a priority objective for the cane industry of Réunion Island at the moment, since this orientation would imply a risk of selecting for lower sucrose content (ERS), given the slightly negative correlation ( -0.13 to -0.36 depending on programmes) between the two traits (Table 3). However, in the local cane industry as in several other sugarproducing countries payment of cane for its fibre content tends to increase because of the usefulness of fibre for electricity production. Any change in cane payment could justify some adaptation in the weights given in the selection to sucrose content and fibre content, respectively. Compared to the usual standards (R577 and R579), results of fibre content pictured in Fig. 2 indicate an important and immediate potential of selection in eRcane programme on this criterion if the need arises. This would be possible without detrimental effect neither on cane yield nor on economic index, given the very low correlation between fibre content and these two traits (Table 3).

\section{Breeding Strategies for Sucrose Yield}

In the present study, cane yield (CY) and sucrose content (ERS) which both determine sucrose yield per unit area showed very frequently non-significant genetic correlations which were almost null in the three programmes (LM, SB and ES) and occasionally slightly negative in one programme only (VB). Such non-significant correlations between CY and ERS have also been observed by other authors (Milligan et al. 1990, 1996; Sandhu and Saini 1997; Baffa et al. 2014; Liu et al. 2016). Therefore, selection pressure exerted on one trait does not affect the other one. Given the primary importance of the objective of selection for $\mathrm{CY}$ and the related economic index (EI), these results justify medium-term efforts on specific genetic improvement in both CY and ERS, in particular for sucrose content (ERS). This trait has high economic value in the sugar industry (Jackson, 2005) since it increases sugar produced from farms and mills with very little increase in marginal costs through harvesting, cane transportation or milling. However, genetic variation of sucrose content among material produced in typical breeding programmes is usually modest as illustrated by $\mathrm{CV}_{\mathrm{g}}$ for ERS found here (Table 2) and also by other authors (Brown and Glaz 2001; Milligan et al. 2003; Neto et al. 2013; Baffa et al. 2014). To increase sucrose content over its current limit, mediumterm breeding strategies based on recurrent breeding strategies are desirable and appropriate. Recurrent breeding approaches for ERS can be effective after a few breeding cycles (Lingle et al. 2010) and can be based on relatively short generation intervals (Kennedy 2005) due to the favourable genetic properties of ERS: moderate-to-high narrow sense heritability (Hogarth 1971; Gravois et al. 1991; Ramdoyal and Badaloo 1998) and good repeatability within and between early stages (Mamet and Domaingue 1999; Jackson and McRae 2001; Bressiani et al. 2003). eRcane has recently engaged a "high ERS" breeding programme based on a recurrent selection scheme with a massive weight given to ERS in the view of supporting enhanced genetic gains in the medium term. The first recurrent cycle has already produced an encouraging substantial increase in one unit of Brix, which correspond to about 0.75 unit of sucrose content (Pers. com. A. ThongChane).

\section{Conclusion}

Considering the high costs and long duration of the Sugarcane Variety Development programmes of Réunion Island, it is important to periodically assess their ability to select competitive genotype populations for sugar yield components. This retrospective study was based on the last decade of variety trials conducted in the most advanced stage of local selection in four regional selection programmes located in different agro-climatic zones of production. This study is the first long-term report on the effectiveness of these regional selection programmes to identify superior genotypes for sugar yield since their routine operation in the early 2000s. Over the last decade, the frequency of candidates exceeding the local control by $120 \%$ for the economic index is common in the two programmes characterized by climatic (VB) or edaphic (SB) stresses and is much rarer in the other two programmes. Genetic gain for the economic index was oriented upwards in all four programmes, and its mean increase per year was highly significant in two of them (LM and VB). All together, these results indicate competitive breeding germplasm and provide trends indicating that potentially high genetic gains could be expected among some varieties to be released for cultivation in the near future. To maintain long-term genetic gains, specific efforts of breeding for sucrose content through recurrent approaches represent a strategic investment.

Acknowledgements The authors wish to thank eRcane technical staff members J.P. Diorflar, G. Morel, F. Poiny, A. Jacquemard and P. Langlois for experimental fieldwork, as well as J.M. Sinama, J. Bernard, M. Zoide and C. Roussel for laboratory determination of quality traits of sugarcane varieties. This study was funded by eRcane Company and by Conseil Regional de la Reunion and European Union (European Regional Development Fund-ERDF) through the grant attributed to Valbiocan project. 
Author Contributions BS managed the variety development program. ATC created the varieties. LB implemented the field experiments. TD and JYH conceived and performed the statistical analyses. BS, ATC, LB, TD and JYH discussed the results. TD and JYH wrote the paper.

\section{Compliance with Ethical Standards}

Conflict of interest The authors declare that they have no conflict of interest

Ethical Approval The authors declare that the experiments presented in this publication comply with current French laws.

Open Access This article is distributed under the terms of the Creative Commons Attribution 4.0 International License ( http://creativecommons.org/licenses/by/4.0/), which permits unrestricted use, distribution, and reproduction in any medium, provided you give appropriate credit to the original author(s) and the source, provide a link to the Creative Commons license, and indicate if changes were made.

\section{References}

Acreche, M.M., J.V. Saez, and J.C. Vera. 2015. Physiological bases of genetic gains in sugarcane yield in Argentina. Field Crops Research 175: 80-86.

Baffa, D.C.F., P.M.A. Costa, G. Silveira, F.J.F. Lopes, et al. 2014. Path analysis for selection of saccharification-efficient sugarcane genotypes through agronomic traits. Agronomy Journal 106(5): $1643-1650$.

Bressiani, J.A., R. Vencovsky, and A.G. Da Silva. 2003. Repeatability within and between selection stages in a sugarcane breeding program. Journal of American Society of Sugarcane Technologists 23: 40-47.

Brown, J.S., and B. Glaz. 2001. Analysis of resource allocation in final stage sugarcane clonal selection. Crop Science 41: 57-62.

Corcodel, L., C. Roussel, and M. Decloux. 2011. Energy content: A new approach to cane evaluation. International Sugar Journal 113: $14-17$.

Cox, M.C., and J.K. Stringer. 2007. Benchmarking genetic gains from new cultivars in Queensland using productivity data. Proceedings of the International Society of Sugar Cane Technologists 26: 624-631.

De Mendiburu, F. 2014. Agricolae: Statistical procedures for agricultural research. $\mathrm{R}$ package version, 1(1).

Edme, S.J., J.D. Miller, B. Glasz, P.Y.P. Tai, and J.C. Comstock. 2005. Genetic contribution to yield gains in the Florida sugarcane industry across 33 years. Crop Science 45: 92-97.

Falconer, D.S., and T.F.C. Mackay. 1996. Introduction to quantitative genetics, 4th ed. Harlow: Longman.

Gravois, K.A., S.B. Milligan, and F.A. Martin. 1991. Additive genetic effects for sugarcane yield components and implications for hybridization. Tropical Agriculture 68(4): 376-380.

Guilly, S., T. Dumont, A. Thong-Chane, L. Barau, and J.Y. Hoarau. 2017. Analysis of multienvironment trials (MET) in the sugarcane breeding program of Réunion Island. Euphytica 213(9): 213.

Hoarau, M. 1969. Sugar cane analysis by hydraulic press method. International Sugar Journal 71: 328-332.

Hogarth, D.M. 1971. Quantitative inheritance studies in sugar-cane I. Estimation of variance components. Australian Journal of Agricultural Research 22: 93-102.
Holland, J. 2006. Estimating genotypic correlations and their standard errors using multivariate restricted maximum likelihood estimation with SAS Proc MIXED. Crop Science 46: 642-654.

Hugot, E. 1958. Critère et formule de comparaisons entre champs, parcelles, variétés ou traitements de cannes. Revue Agricole et Sucrière de l'Ile Maurice 37: 212-216.

Jackson, P.A. 2005. Breeding for improved sugar content in sugarcane. Field Crops Research 92: 277-290.

Jackson, P.A., and T.A. McRae. 2001. Selection of sugarcane clones in small plots: Effects of plot size and selection criteria. Crop Science 41: 315-322.

Kang, M.S., J.R. Sosa, and J.D. Miller. 1990. Genetic variation and advance for rind hardness, flowering and sugar yield traits in sugarcane. Field Crops Research 23: 69-73.

Kang, M., J. Miller, P.Y.P. Tai, J. Dean, and B. Glasz. 1987. Implications of confounding of genotype $\times$ year and genotype $\times$ crop effects in sugarcane. Field Crops Research 15: 349-355.

Kennedy, A.J. 2005. Breeding improved cultivars for the Caribbean by utilisation of total biomass production. Proceedings of the International Society of Sugar Cane Technologists 25: 491-499.

Littell, R., G. Milliken, W. Stroup, R. Wolfinger, and O. Schabenberger. 2006. SAS for mixed models, 2nd ed. USA: SAS Institute Inc Cary NC.

Lingle, S.E., R.M. Johnson, T.L. Tew, and R.P. Viator. 2010. Changes in juice quality and sugarcane yield with recurrent selection for sucrose. Field Crops Research 118: 152-157.

Liu, J., J. Basnayake, P.A. Jackson, X. Chen, J. Zhao, P. Zhao, L. Yang, et al. 2016. Growth and yield of sugarcane genotypes are strongly correlated across irrigated and rainfed environments. Field Crops Research 196: 418-425.

Lynch, M., and B. Walsh. 1998. Genetics and analysis of quantitative traits. Sunderland, MA: Sinauer Associates, Inc Publishers.

Mamet, L.D., and R. Domaingue. 1999. Shortening the selection process for sugarcane. Experimental Agriculture 35: 391-405.

Milligan, S., K.A. Gravois, K.P. Bischoff, and F.A. Martin. 1990. Crop effects on genetic relationships among sugarcane traits. Crop Science 30: 927-931.

Milligan, S.B., K.A. Gravois, and F.A. Martin. 1996. Inheritance of sugarcane ratooning ability and relationship of younger crop traits to older crop trait. Crop Science 36: 45-50.

Milligan, S.B., M. Balzarini, and W.H. White. 2003. Broad-sense heritabilities, genetic correlations, and selection indices for sugarcane borer resistance and their relation to yield loss. Crop Science 43: 1729-1735.

Neto, H.Z., E. Daros, J.C. Bespalhok Filho, C.A. Scapim, M.C.G. Vidigal, and P.S. Vidigal Filho. 2013. Selection of families and parents of sugarcane (Saccharum spp.) through mixed models by joint analysis of two harvests. Euphytica 193(3): 391-408.

Ramdoyal, K., and G. Badaloo. 1998. Inheritance of agronomic traits in commercial hybrid sugar cane populations in contrasting environments and in different crop cycles. Journal of Genetics \& Breeding 52: 361-368.

Roussel, C., A. Warembourg, F. Davrieux, J. Bernard, and C. Poser. 2015. Development of NIRS method for routine assessment of sugarcane quality in Réunion Island. Abstract In: Pushing the frontiers of sugarcane improvement, 11th Germplasm \& Breeding and 8th Molecular biology ISSCT Workshop. eRcane, SaintGilles, Réunion, p. 16.

Sandhu, D., and G.S. Saini. 1997. Inter-relationships among cane yield and commercial cane sugar and their component traits in autumn plant crop of sugarcane. Euphytica 95(1): 109-113.

Saranin, A.P. 1986. The press method of sugarcane analysis. Proceedings of the Australian Society of Sugarcane Technologists 8: 33-42. 
Steel, R., and J. Torrie. 1980. Principles and procedures of statistics. A biometrical Approach. Singapore: McGraw Hill Editions.

Tena, E., F. Mekbib, and A. Ayana. 2016. Heritability and correlation among sugarcane (Saccharum spp.) yield and some agronomic and sugar quality traits in Ethiopia. American Journal of Plant Sciences 7: 1453-1477.
Zhou, M.M., and E.T. Gwata. 2016. Quantifying sugarcane cultivar genetic gains in the Midlands region of South Africa. Agronomy Journal 108(1): 342-348.

Publisher's Note Springer Nature remains neutral with regard to jurisdictional claims in published maps and institutional affiliations. 